\title{
Thinking Aloud: How Nurses Rationalize Responses to Monitor Alarms
}

\author{
Andrew S Kern-Goldberger, MD ${ }^{1,23^{*}}$, Veronica S Zielinski, MSN, RN, CPN², Christopher P Bonafide, MD, MSCE ${ }^{3,4}$
}

'Division of Child and Adolescent Health, Department of Pediatrics, Columbia University Irving Medical Center, New York, New York; ${ }^{2}$ New York-Presbyterian Morgan Stanley Children's Hospital, New York, New York; ${ }^{3}$ Section of Hospital Medicine, Children's Hospital of Philadelphia, Philadelphia, Pennsylvania; ${ }^{4}$ Perelman School of Medicine at the University of Pennsylvania, Philadelphia, Pennsylvania.

$\mathrm{n}$ the past five years, it has become increasingly apparent that hospital physiologic monitoring systems are not functioning optimally for children. On pediatric wards, $26 \%-48 \%$ of children are continuously monitored, and these children generate between 42 and 155 alarms per day. ${ }^{1}$ Just $1 \%$ or fewer are considered actionable or informative, slowing nurses' response times and placing patients at risk of delayed recognition of life-threatening events. ${ }^{2,3}$ While some factors associated with alarm response times have been elucidated, ${ }_{1}{ }^{3}$ in order to design safe and effective monitoring systems, further work is needed to understand the complex decision-making process that nurses face when encountering alarms outside a patient's room. It is in this area that Schondelmeyer and colleagues strive to enhance our understanding in this issue of the Journal of Hospital Medicine. ${ }^{4}$

Schondelmeyer et al. conducted a single-center, observational study using mixed methods in a general pediatric unit. Trained observers shadowed nine nurses one to four times each, during which nurses were asked to "think aloud" as they managed physiologic monitor alarms, rationalizing their decisions about how and why they might respond for the observer to document. Observers accumulated 61 patient-hours of observation before investigators halted data collection because new insights about alarm responses were no longer emerging from the data (thematic saturation).

Nurses thought aloud about 207 alarms during the study, which the investigators estimated comprised about one third of the alarms that occurred during observation periods. Most of the 207 occurred while the nurse was already in the patient's room, where a response decision is uncomplicated. More interesting were the 45 alarms heard while outside the patient's room, where nurses face the complex decision of whether to interrupt their current tasks and respond or delay their response and assume the associated risk of nonresponse to a potentially deteriorating patient. Of the 45 alarms, nurses went into the room to evaluate the patient 15 times and, after doing so, reported that five of the 15 warranted in-person responses to address technical issues with the monitor, clinical issues, or patients' comfort. Reassuring clinical contexts-such as presence of the medical team or family in the

*Corresponding author: Andrew S. Kern-Goldberger, MD; E-mail: kerngoldba@email.chop.edu; Telephone: 215-590-1214

Received: May 22, 2019; Accepted: May 24, 2019

๑ 2019 Society of Hospital Medicine DOI 10.12788/jhm.3254 room and recent patient assessments-were the reasons most commonly provided to explain alarm nonresponse.

This study has two key limitations. First, the authors designed the study to observe nurses' responses until thematic saturation was achieved. However, the small sample size (nine nurses, 45 out-of-room alarms) could raise questions about whether sufficient data were captured to make broadly generalizable conclusions, given the diverse range of patients, families, and clinical scenarios nurses encounter on an inpatient unit. Second, by instructing nurse participants to verbalize their rationale for response or nonresponse, investigators essentially asked nurses to override the "Type 1", heuristic-based reasoning ${ }^{5}$ that research suggests regulates nursing responses to alarms when adapting to circumstances requiring high cognitive demand or a heavy workload. ${ }^{3}$ While innovative, it is possible that this approach prevented the investigators from fully achieving their stated objective of describing how bedside nurses think about and act upon alarms.

Nonetheless, the findings by Schondelmeyer and colleagues extend our emerging understanding of why alarm responses are disconcertingly slow. Nursing staff's dismissal of monitor alarms that are discordant with a reassuring patient evaluation underscores the imperative to reduce nuisance alarms. Furthermore, the explicit statements justifying alarm nonresponse because of the presence of family members build upon prior findings of longer response times when family members are at the bedside ${ }^{3}$ and invite a provocative question: how would family members feel if they knew that they were being entrusted as a foundational component of safety monitoring in the hospital? In their recently published study conducted at the same hospital, ${ }^{6}$ Schondelmeyer's team elicited perceptions that families are deeply concerned about staff nonresponse to alarms-as one nurse stated, parents "wonder what's going on when no one comes in." While there is a valuable role for integrating families into efforts to overcome threats to patient safety, as has been achieved with family error reporting ${ }^{7}$ and communication on family-centered rounds, ${ }^{8}$ this must occur in a structured, explicit, and deliberate manner, with families engaged as key stakeholders.

In summary, while Schondelmeyer and colleagues may not have exposed the depth of implicit thinking that governs nurses' responses to alarms, they have highlighted the high-stakes decisions that nurses confront on a daily basis in an environment with exceedingly high alarm rates and low alarm actionability. The authors cite staff education among potential solutions to improve the safety of continuous monitoring, but such an inter- 
vention cannot be effective in a system that places impossible burdens on nurses. An openly family centered and multidisciplinary approach to reengineering the system for monitoring hospitalized children is needed to enable nurses to respond quickly and accurately to patients at risk of clinical deterioration.

Disclosures: The authors report no conflicts of interest.

\section{References}

1. Schondelmeyer AC, Brady PW, Goel W, et al. Physiologic monitor alarm rates at 5 children's hospitals. J Hosp Med. 2018;13(6):396-398. https://doi. org/10.12788/jhm.2918

2. Bonafide $\mathrm{CP}$, Lin $\mathrm{R}$, Zander $\mathrm{M}$, et al. Association between exposure to nonactionable physiologic monitor alarms and response time in a children's hospital. J Hosp Med. 2015;10(6):345-351. https://doi.org/10.1002/jhm.2331.
3. Bonafide $\mathrm{CP}$, Localio $\mathrm{AR}$, Holmes $\mathrm{JH}$, et al. Video analysis of factors associated with response time to physiologic monitor alarms in a children's hospital. JAMA Pediatr. 2017;171(6):524-531. https://doi.org/10.1001/jamapediatrics.2016.5123

4. Schondelmeyer A, Daraiseh NM, Allison B, et al. Nurse responses to physiologic monitor alarms on a general pediatric unit. J Hosp Med. 2019;14(10):602-606. https://doi.org/10.12788/jhm.3234.

5. Croskerry P. A universal model of diagnostic reasoning. Acad Med. 2009;84(8):1022-1028. https://doi.org/10.1097/ACM.0b013e3181ace703.

6. Schondelmeyer AC, Jenkins AM, Allison B, et al. Factors influencing use of continuous physiologic monitors for hospitalized pediatric patients. Hosp Pediatr. 2019;9(6):423-428. https://doi.org/10.1542/hpeds.2019-0007.

7. Khan A, Coffey M, Litterer KP, et al. Families as partners in hospital error and adverse event surveillance. JAMA Pediatr. 2017;171(4):372-381. https://doi. org/10.1001/jamapediatrics.2016.4812.

8. Khan A, Spector ND, Baird JD, et al. Patient safety after implementation of a coproduced family centered communication programme: multicenter before and after intervention study. BMJ. 2018;363:k4764. https://doi. org/10.1136/bmj.k4764 